betrug durchschnittlich $95 \%$ der Theorie. Die angewandte Stromdichte läßt sich natürlich noch erhöhen, wenn man die Walze rascher rotieren läßt. Die abgezogenen Kupferrohre hatten eine Wandstärke von $5 \mathrm{~mm}$ und zeigten eine glatte, glänzende Oberfläche ohne knospige Auswüchse. Nach Versuchen der Kgl. mechanisch-technischen Versuchsanstalt in Charlottenburg zeigten die aus dem Bade entnommenen Rohre eine Zugfestigkeit, die anderthalbmal so groß war, als den Anforderungen der Kaiserlichen Marine entspricht. Dieselbe läßt sich jedoch auf mindestens das Doppelte erhöhen, wenn man die Rohre cinem Nachziehen unterwirft. In der gleichen Weise, wie beschrieben, lassen sich auch profilierte Kupferrohre herstellen, wenn man eine entsprechend geformte Unterlage wählt. Na-

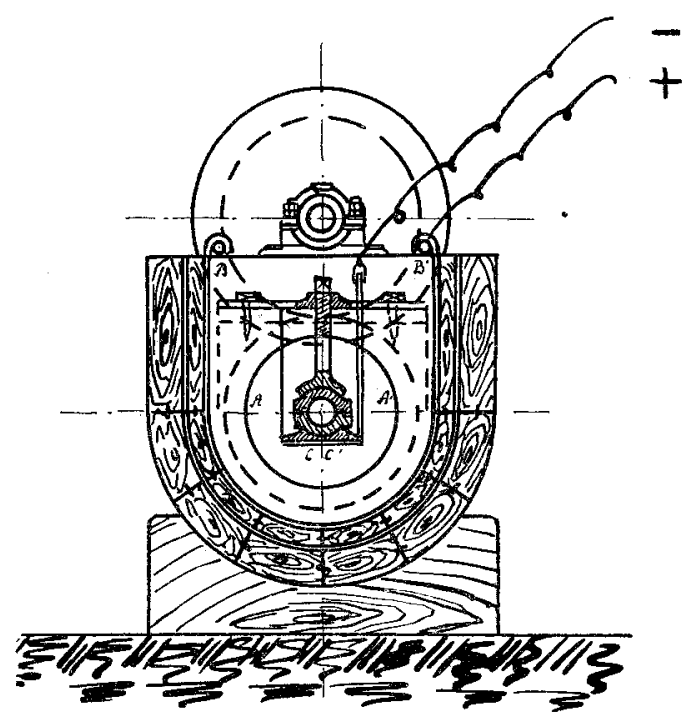

türlich ist man nicht an die hier gegebenen GröBenverhältnisse gebunden, sondern es ist auch die Herstellung von Rohren beliebiger Dimensionen, nach den seitherigen Versuchen zu urteilen, ohne größere Schwierigkeiten möglich.

Die Versuche ergaben die große Uberlegenheit dieses Verfahrens gegenüber allen anderen für diesen Zweck bekannten Herstellungsweisen. Nicht allein, daß es gestattet, glatte und dichte Kupferniederschläge bei geringer Rotationsgeschwindigkeit $\mathrm{zu}$ erhalten, sondern, daß es ein Kupfer von besonders hervorragenden Eigenschaften liefert. Die scheuernde Wirkung des Kieselgurs auf das niedergeschlagene Kupfer ist eine so vorzügliche, daB sämtlicher Wasserstoff entfernt wird, wodurch es keine der bei den üblichen Verfahren sich zeigenden Nachteile aufweist. Es eignen sich deshalb derartige Rohro für alle Zwecke; besonders aber werden sie vorteilhafte Anwendung finden, wo es sich darum handelt, Kupferrohre anzuwenden, die auf hohen Druck beansprucht werden sollen.

Da sich das genannte Verfahren nicht nur auf die elektrolytische Abscheidung des Kupfers beschränkt, so dürfte dasselbe auch bei der Elektrolyse solcher Metalle, die leicht lockere und poröse Niederschläge liefern, mit gleichem Erfolg verwendet werden.

\section{Über Katalyse.}

Von G. Bredit, Heidelberg.

(Eingeg. d. 4./1, 1907.)

\section{I. $\mathrm{Z}$ u r A bwe hr.}

Wenn ein auf gewissen Gebieten mit Recht hoch angesehener Chemiker wie $\mathrm{R}$ as chig, wicd rrholt auf anderen, ihm weniger bekannten Pfaden, o h $\mathrm{n}$ e sich vorher genügend zu instruieren. ,den Mund so weit a ufmacht", (die in Anführungszeichen stehenden Ausdrücke stammen von $R$ as c h i g), daß er ohne hinreichende Gründe einen Grundpfeiler der physikalischen und chemischen Atomistik, wie dic A v o g a d r o sche Regel (vgl. diese Z. 18, 1283, 1298 [1905]) für morsch exklärt, oder daß er einem wissenschaftlich gebildeten Hörer- und Leserkreise, wie dem Verein deutscher Chemiker, in feierlicher Versammlung zumutet, seine nunmehr genügend (diese Z. 19, 1985 u. 2049 [1906]) charakterisierten Spekulationen für die Quintessen\% der Arbeit einer Reihe großer „Meister" (diese Z. 19, 1761 [1906]) tatenreicher Jahrhunderte und des heutigen Standes der atomistischen und katalytischen Forschung zu halten, daß er, wie R a s chig (diess Z. 19, 2084-2085 [1906]), den Anspruch macht, ,S ch 1 üs se, welche schon viele Fachgenossen, wenn a u h meist unbew u $\mathrm{n}, \mathrm{dunkel}$ und unklardarausgezogen hatten, klar und in einigermaBen straffer Folgerichtigkeit" zu ordnen, dann kann man ihn nicht hinter die Immunität einer Fostreds ${ }^{1}$ ) verschanzen, und er darf sich nicht beklagen, wenn die Kritik, mit der ich durchaus nicht allein stehe, prüft, was.denn nun eigentlich Gediegenes an der Sache ist, auch wenn sie bei volls t ä n d i g n e gativem Resultat etwas herb ausfällt. Der Ton der guten alten Zeit (l. c. S. 2086), den Ras chig herbeisehnt, ist übrigens gelegentlich doch sehr erheblich schärfer gewesen, wie $\mathbf{R}$ a s $\mathrm{c} \mathrm{h}$ ig sich leicht durch Lektüre seines Gewährsmannes L i e b i g und anderer in ihren Polemiken gegen sehr verdiente Leute überzeugen kann $^{2}$ ). Für $\mathbf{R}$ a s c h i gs eigentümliche Vernichtung der A v o g a d r o schen Regel, zu der ich früher als Zuhörer nicht gerade aus Zustimmung geschwiegen hatte, hat sich bereits von berufener Seite ${ }^{3}$ ) die Charakterisierung eingefunden, in seinem Nürnberger ,K a r t e n h a u s" (1. c. S. 2083) aber will der Erbauer trotz meinem und $\mathrm{L} u t h$ ers Abraten wohnen bleiben.

Herr $R$ as chig erwidert den 'Ton meiner Kritik, der ihm nicht gefällt, zum mindesten nicht mit einem besseren und hat es $u$. a. für passend gefunden, meine persönliche Stellung als Leh rer (l. e. S. 2083) zu kerühren Er beklagt sich darüber,

1) A n m e r k u g: Als Muster einer g e is t reichen, phantasievollen, aberstets exakten, atomistisehen Festplauderei sei Meister H. K o p p s köstliche Broschüre: ,Aus der Molekularwelt"c, (Heidelberg 1882) empfohlen, die diese Zierde des Heidelberger ,Lehrkörpers" dem Altmeister B u n s e n, als dessen Schülẹr sich uns R a s ch i g (diese Z. 19, 2085 ([1906]) vorstellt, zu seinem Geburtstage gewidmet hat.

2) Vgl. z, B. L i e b i g s Ann. 2, 19 (1832); 25, 29 (1838); 33, 308 (1840); 5\%, 105, 112 (1846).

3) Lu nge u. B erl, diese Z. 19, 881 (1906). 
daß ich als soleher ihm oder gleichdenkenden Lesern mit meinen Ausführungen nicht das richtige Verständnis habe beibringen können. Wenn jemand aber eine solche Klage gegen einen Lehrer erhebt, so mag der unparteiische Leser auch prüfen, ob denn in diesem Falle die Schuld allein beim ,Lehrer" liegt. Wie man aus meinem Aufsatze leicht ersehen konnte, hatte ich es mich in der Tat (diese Z. 19, 1985 [1906]) einige Mühe kosten lassen, Herm $\mathrm{R}$ a schig auf die vielen wunden Stellen seiner Beweisführung hinzuweisen, und i $h \mathrm{~m}$ in $\mathrm{z}$ a $\mathrm{h} l$. reichen Zitaten genaudie Quellen a $n g$ e $g$ e ben, wo er das vermißte, richtige Verstiandnis bei vorurteils losem Studium gefunden hätte. DaB er meine Kritik und jene Quellen, die ich doch unmöglich alle wörthich abdrucken konnte, nicht aufmerksam genug gelesen hat, muB ich aus zahlreichen i r r i g e $\mathbf{n}$ Un t e r stellungen entnehmen, dis mir Raschig in seiner allzuschleunigen Entgegnung macht. So versichert er den Leser z. B., nach meinen Angaben habe nur ,,e in Forscher" bisher einen Einfluls eines magnetischen Feldes auf die Reaktionsgeschwindigkeit nicht feststellen (l. c. S. 2085 unten) können. Tats ä chli $\mathrm{ch}$ habe ich ihm aber (l. c. S. 1987, Fußnote 26) z w e i g a $\mathrm{n}$ verschieden e Arbeiten voneinander unabhängiger Forscher genannt; ich habe auch nicht behauptet, daß jener Einfluß nicht ex is tie $\mathrm{r} t$, sondern $\mathrm{S} p \mathrm{e}$. $\mathrm{k}$ u 1 a t i o n e n darüber als bisher ex perim en tell ergebnis los gekennzeichnet.

,Das Stärkste aber ist" (1. c. S. 2085 oben) R aschigs Entrüstung daß ich unserem Leserkreise ein Elementarbeispiel aus, d e r E l e k trizitätslehre" (das übrigens gar nicht voa mir herrührt) gebracht hätte, was jedoch nur geschah, um ihm an Stelle, einer trockenen physikalisch-chemischen Abhandlung" (1. c. S. 2084 unten) an diesem bekannten Gleichnis den Begrift hintercinander geschalteter chemiseher $W$ i d e r $\mathrm{s} \ddot{a} \mathrm{nde} z \mathrm{zu}$ veransehaulichen. Diesen letzteren Begriff soheint $R$ as eh ig noch immer nicht zu kesnen, denn er hat sich, wie aus sciner Entrüstung über die vermeintlichen, $A \mathrm{n}$ fan $\mathrm{gs}$. gründe der Elektrizit ̈̈tslehre" hervorgeht, noch immer nicht ans den von mir an (1. c. S. 1987, Furnoten 27 u. 28) jener Stelle deutlich angegebenen Quellen ${ }^{4}$ ) unterrichtet, und daher hat er das dort klar zutage liegende Tertium comparationis in diesem Gleichnis gar $\mathbf{n i c h t}$ einmal gesehen. Raschig merkt es in seinem Eifer gar nicht einmal, da $\beta$ erdurch meine von ihm verpönte „, Logik" (1.c. S. 2085), ohne es z u wissen und zu wollen, zu meiner Freudein der That zu demselben richtigen Sehlussegeführtworden ist, aufden ich ihn wirklich gerade durch das Gleichnis bringen wollte: da $B$ ämlich zwei hintereinander geschaltetestufenreaktionen, wenn nur ihre chemischen Widerstände

4) Nernst, Theoret. Chemie (4. Aufl.) S. 656. $\mathrm{S}$. auch $\mathrm{Ostw}$ a $1 \mathrm{~d}$, Lehrb. d. allgem. Chemie, 2. Aufl., II (2), 200; v a n 't $\mathrm{H}$ of f , Vorlesungen I, 181. „Besonders lehrreich ist auch die schöne Darstellung in Ostwalds Leitlinien der Chemie, Ieipzig 1906 , S. 302,307 , sowie S. 288 . * in s u m m a kleiner sind als der chemische Widerstand der sogenannten, direkten" Reaktion ta $\mathrm{t} \mathrm{s} a \mathrm{ch} \mathrm{li} \mathrm{ch}$ (gerade so wie die beiden hintereinander geschalteten kleinen elektrischen Widerstände) e be n a $\mathrm{u} \mathrm{ch}$ kein en $\mathrm{U} \mathbf{m}$ we g, sondern wirklich den direkteren W e g für den Energieausgleich b ed e u ten. Bei etwas längerem Nachdenken und Studium der genannten Quellen wird $\mathrm{R}$ as e h i g also wohl endlich einsehen, daß ich hier als , Lehrer" mein Gleichnis durchaus richtig angewandt und a $\mathrm{uch}$ i h $\mathrm{n}$, ohneda $\beta$ eresfreilich merkte, zum richtigen Schlusse gezwungen habe. Ebenso behauptet (l. c. S. 2083) R as c hig fäls ch lich, ich hätte nicht angegeben, wie ich mir den Unterschied der Keim. w i r k u g e n bei der Krystallisation $v$ on de $n$ gew öhnlichen Katalysen denke. Den von mir an der betreffenden Stelle (1. c. S. 1986, Fußnote 15) genannten Aufsatz in den „Ergebnissen der Physiologie" hat also Rasehig, be vor er antwortete, ebenso wenig mit Erfolg gelesen, wie die betreffende Stelle in $\mathrm{Ostw}$ alds Vortrag ( $\mathrm{S}$. 7), denn aus beiden geht der gesuchte Unterschied klar hervor. Bei $\mathrm{O}$ s tw a ld findet jeder aufmerksame Leser, daß eine überkühlte Schmelze ohne Zusatz des festen Keimes ein in sich stabiles Gebilde ist, während z. B. eine $\mathrm{H}_{2} \mathrm{O}_{2}$-Lösung oder Methylacetat lösung sich auch schon ohne Zusatz eines Katalysators, wenn auch viel langsamer, zersetzt. In meiner von Herrn $\mathbf{R}$ as $\mathrm{ch}$ i g nicht gelesenen $\mathrm{Ab}$ handlung hatte ich gezeigt, daß bei der Keimwirkung die erzeugte Geschwindigkeit unabhängig von der Menge des auslösend zugesetzten Stoffes (in überkühlten Schmelzen) gemacht werden kann, daß bei der gewöhnlichen Katalyse (z. B. Zuckerinversion) dagegen die erzeugte Geschwindigkeit im allgemoinen von der Menge des Katalysators abhängt. Zuzugeben ist allenialls, daß bei $\mathrm{Ost}$ w a $1 \mathrm{~d}^{5}$ ) die Abtrennung beider Erscheinungsgruppen für $\mathrm{R}$ a s chig nicht genügend scharf herausgearbeitet gewesen ist, ebenso wie ich mit meinem Lehrer Ostwald, wenn er wirklich das Gleichnis rom Funken im Pulverfaß in dem von $R$ a s c h ig gemeinten Sinne gebraucht haben sollte, doch durchaus nicht immer absolut übereinstimmen $m \mathbf{u} \mathbf{B}$, wie mir Raschig merkw ür igerweise (l. c. S. 2084 oben) z u mutet. Wenn ich nicht immer unbedingt in verba magistri schwöre, so wird mir dieser, wie ich ihn kenne, das am wenigsten verübeln. Ich vermute aber immerhin, daß Ostwald überhaupt nicht die Te m peraturerhöhung und die dadurch bedingte unklare Raschigsche ,Formänderung der Moleküle" durch den Funken, sondern lediglich die Reaktionsbesch leunigung durch einen fremden Zusatz in Parallele zur Wirknng eines Katalysators setzen wollte.

Es ist natürlich unmöglich, hier die zahlreichen Punkte, welche $\mathrm{R}$ as ch ig mit seiner bekannten staunenswerten Dialektik zu versehieben sucht, alle richtig zu stellen: Als Beispiel führe ich nur an, daß in seiner Entgegnung $R$ as chig lung.

) Im Gegensatz zu meiner genannten Abhand- 
jetzt plötzlich (1. c. S. 2084) nicht an ,glatt, poliert, glänzend ...." bei dem Einfluß der Oberfläche gedacht haben will, während er in $\mathrm{N}$ ü $\mathrm{r} n$ berg den Spring schen Versuch mit der polierten bzw. geritzten Platinschale ins Treffen (l. c S. 1763) führte. Wenn er mir unterstellt, ich hätte das Schmelzen des Schwefels bei der Explosion des Schießpulvers geleugnet, so ,geht das über meinen Horizont" (Raschig l. c. S. 2085 unten). Uber die vielen Einwände, denen $R$ as ehigs hypothesenstrotzende Arbeiten mit ibrem oft sehr problematischem, wenn auch, wie immer, sehr schön vorgetragenem ${ }^{6}$ ) Material unterliegen, könnte man Bände schreiben. Die geschichtliche Entwickelung pflegt aber derartige Dinge von selbst auszuscheiden und ich babe daher nicht die Absicht, mich ohne Not weiter damit zu beschäftigen. Den Mund werde ich aber trotz Herrn $\mathbf{R}$ as chig stets auftun, wenn mir die Gefahr der Mythenbildung auf einem auch von mir ein wenig bebauten Gebiete zu drohen scheint, und im Interesse der Wahrheit werde ich es stets ablehnen, das, was mir schwarz erscheint, weiß zu nennen.

II. Die Fragenach dem Einflusseder ,Formänderung der Moleküle" bei der Katalysehat einen experimontellen $\mathrm{sinn}$.

Jetzt muB ich Herrn R as chig eine Überraschung bereiten: Ich bin nämlich (als Schüler nicht allein $O s t w$ a $l d s$, sondern auch $v$ an' $t$ Hoffs (und wohl auch ein wenig aus eigenen Gründen) bis zu einem gewissen Grade Atomist ${ }^{7}$ ) allerdings nicht in $\mathrm{R}$ as $\mathrm{eh}$ igs Sinne. Ich habe auch in meiner Kritik nirgends die ,Atzlauge des Hohnes" (1. c. S. 2085) über die Gedankenwelt eines $\mathrm{Dalton}$ und Kekulé, wie Raschig mir unterstellt, ",mit billigen Scherzen ausgegossen", denn die Sache war mir viel zu ernst. D i eser Welt verdanken wir ja unsere $q$ u a $\mathrm{nt}$ it a $\mathrm{t}$ ive Veransehaulichung der Stöchiometrie und die großartige Entwicklung der q u a n t i t a t i v e n Lehre von der chemischen Isomerie, von den Benzolderivaten und der Strukturehemie überhaupt. I $\mathbf{n}$ d e $r$ Gedankenwelt $R$ aschigs aber habe ich nichts a ch nur annähernd Ähn. lichos finden können, obwohl er auf jene Vorbilder sich beruft. I ch habe deshalb ganz bew 3 t von jenerechten Ato. mistik in meiner Kritik eine Pseudo. a tom istik unterschieden, welche auch nicht im geringsten, nicht einmal qualitativ, Erscheinungen so voraussagen läßt und quantitativ darstellt, wie es jene echte Atomistik von Anbeginn an getan hat. Ich muß $R$ as e hig nooh mehr gestehen: Ich beschäftige mich nämlich selbst seit einigen Jahren bereits mit dem Ein-

6) Vgl. Lu n g e, diese Z. 17, 1659 (1904)

7) Zu den Gebieten, welche zu einer geläuterten Molekulartheorie u. Atomistik führen dürften, scheint mir außer den von $L u t h$ e r genannten auch die Krystallographie zu gehören. Vergl. van't Hoff, Vorl. II, 148, sowie die schönen Arbeiten von V. G o ld s c h mid t über Lösungskörper, Neues Jahrb. f. Mineralogie Beilage Bd. 17, $355 ; 18,335 ; \mathrm{H}$. B a u m a u er, Die neuere Entw. d. Kristallographie. Braunschweig 1905. flusse der Formänderung der Molek ülo auf die chemische Reaktionsgeschwindigkeit, aber von dem Prinzip eines De Candolle ausgehend: ,da $B$ die Grenze zwischen dem Bekannten und dem Unbekannten niemals so deutlich wahrgenommen wird, wie durch die exakte Beobachtung von $\mathrm{T}$ a $\mathrm{t}$ sa chen, sei es wie die Natur sie unmittelbar bietct, sei es im künstlich angestellten Experiment". Freilich gehe ich dabei von anderen Gesichtspunkten aus und mit ganz anderen Methoden vor als $\mathbf{R}$ a. schig mit seiner Festrede, ,auf dem Wege des logischen Denkens", (1. c. S. 2085). Ich bleibe lieber bei der guten alten, trockene $\mathrm{n}^{4}$ Methode, wie wir sie in ,r e ch ne nd e $n^{6}$ physikalisch-chemischen Abhandlungen $m$ it $m \ddot{g}$. lichst viel exaktem experimentel. le $\mathbf{n} \mathrm{Inh}$ a $1 \mathrm{t}$ finden.

Ein kleiner Anfang dazu ist bereits gedruckt. So habe ich mit Herrn $W$ e in $m$ a y e $r^{8}$ ) experimentell die Frage bearbeitet, wic dünn eine Quecksilberschicht werden muß, bis man ihre kataly tische Wirkung nicht mehr merkt. Das allerdings kleinen Einwendungen noch zugängliche, aber im großen ganzen wohl richtige Resultat war in dor Tat die sogen. Mo. lek u lardimension.

Aber noch von einer anderen Seite war für mich seit langer Zeit vor $\mathbf{R}$ as $\mathbf{c h i g} d$ as Problom der Formänderung der Moleküle einladend $z$ experimenteller Forschung: Nach E. Fischer benehmen sich nämlich die $\mathrm{Enz}$ y $\mathrm{me}$, diese biologisch so wichtigen Katalysatoren, so, als ob sie ron den stereoisomeren rechts- und links-,,Formen der Moleküle" ihres Substrates, z. B. der Zuckerarten, die eine vor der anderen bevorzugen, und $\mathrm{E}$. $\mathrm{Fischer,}$ Ostwal $\mathrm{d}^{9}$ ) u. a. haben diese Spezifität der Fermente vermutungsweise darauf zurückgeführt, da $B$ die En zy me selbst asymmetrische Gebilde seien. Dies hat bei mir vor längerer Zeit den Wunsch erregt, solche Fälle künstich nachzuahmen, in dem ich die Wirkung eines bekann. ten optisch aktiven Katalisators auf die d-Form mit derauf diel-Form eines ebenfalls. optisch aktiven Substrates vergleichen, also gewissermaßen jene Spezifitat der Fermente an einem einfacheren Falle der Katalyse nachahmen wollte. Hierzu liegt ein kle in er experi menteller Versuch in einer unter meiner Leitung von Herrn R. W. B a l co m ${ }^{10}$ ) ausgeführten Dissertation seit einem Jahre gedruckt vor: Die Geschwindigkeit der Zersetzung der optiseh-aktiven Camphocarbons äurein Campher und Kohlendi oxyd wird nämlich, wie $B$ a lo o m festgestellt hat, durch verschiedene L $\ddot{\rho}$. sungsmittel bei derselben Tempe. ratur ganz verschicden beschleu-

8) Ann. d. Phys., Bolt z m a n n-Festschrift $1904,839$. 1902.

9) Uber Katalyse, Vortrag, Seite 31, Leipzig

1ii) Dissertation, Heidelberg 1905. Die Arbeit wird demnächst ausführlicher in anderer Stelle erscheinen. 
nig t. Für andere Fälle hatte dies bekanntlich Menschutkin bereits längst (Z. f. phys. Chem. 6, 41 [1890]) anschaulich festgestellt. 11) Man faßt aus solchen Gründen schon lange auch die Wirkung des Lösungsmittels als eine katalytische auf. Es liegt kein Grund vor, warum in einem und demselben optisch inaktiven Lösungsmittel die d- und die 1-Form der obigen Säure verschieden schnell zerfallen sollten, aber einem o $\mathrm{p}$ t i s $\mathrm{ch}$ - a k t i v e $\mathrm{n}$ Lösungsmittel gegenüber verlangt bekanntlich die stereochemischè 'Theorie ${ }^{12}$ ) und auch Landolts Auffassung der Lösungen, daß die d-Form des Moleküls obiger Säure sich anders benimmt als die l-Form. Wir haben daher bereits lange vor Raschigs Publikation guantitative Versuche gemacht, ob eine, ,Umstülpung" des M o le k üls (diese $Z$. 19, 2086 [1906]), um mit diesem zu reden d. h., wenn wir die d-Form der Säure bei Lösungen in optisch-aktivem Limonen ersetzen durch ihre l-Form, ob als o diese einfachste, Formänderung des Moleküls"eine Änderung der Reaktionsgeschwindigkeit bewirkt. Das Re

11) A n m e rkung: Ich bin mit mejnem Kollegen K n o e v e $\mathrm{n}$ a g e 1, Berl. Berichte 36,2843 (1903), welchen $R$ aschig anführt, durchaus einverstanden, daß alles in der Chemie unter dem Einflusse der Katalyse steht. Schon der Einfluß des Lösungsmittels ist $\mathrm{z}$. B. ein solcher. Das hatte aber bereits mehrere Jahre vor ihm $O \mathrm{~s} t \mathrm{w}$ ald in seinem bekannten Grundriß der allgemeinen Chemie (2. Aufl., Seite 303 u. 515 [1899]); siehe auch O s t wa lds Vortrag: Uber Katalyse, Leipzig 1902, Seite 14-15) so klar ausgesprochen, daß es jedem Arbeiter auf diesem Gebiete geläufig war. Es ist aber doch wohl nicht ganz gleichgültig, wie auch $\mathrm{K} n$ o e ren a g e l zugibt, ob dieser Einfluß auf die Reaktionsgeschwindigkeit $z$. B. bei einem fremden Zusatze von 0,0001\% kaum merklich ist, oder nur wenige Prozente oder das Millionenfache der ursprïnglichen Geschwindigkeit beträgt. Im letzteren Falle dürfen wir also doch sehr wohl aus der Katalyse eine besondere Gruppe von Erscheinungen machen, wie etwa aus der organischen Chemie, aus der Gleichgewichtslehre, der Elektrochemie, obwohl auch diese, natürlich ebenso wie die Kontaktchemis, den a $11 \mathrm{~g}$ e m e i n e n chemischen Gesetzen mit unterliegen. Soll man z. B. etwa deshalb eine Lehre von der Strahlung oder von der Elektrizität ablehnen, weil schließlich alles mehr oder weniger strahlt und elektrisch ist? Und ebenso wie die Strahlung oder die Elektrizitätsentwicklung sehr verschiedene Ursachen, z. B. thermische, chemische, mechanische usw, haben kann, so wird es auch bei der Katalyse sein. Die Frage, wo man die Grenze für das Gebiet der Katalyse ziehen solle, halte ich daher aus demselben Grunde für nebensächlich, wie die einer starren Abgrenzung der Lehre von der Strahlung oder Elektrizität von der übrigen Physik. Alle Katalysen nach demselben Schema erklären zu wollen, hat kein einsichtiger Physikochemiker jemals versucht, im Gegenteil bringt die fortschreitende Erkenntnis immer feinere Differenzierungen hervor, und gerade das ist der Vorteil der spezialisierten katalytischen Forschung, die das Allgemeine nicht aus dem Auge läßt und doch nicht in jedem Falle $n$ u $r$ ein spezielies anorganisches oder organisches Problem sieht.

12) Vgl. va n't $\mathrm{H}$ of $\mathrm{f}$, Lagerung der Atome im Raume (1894) Seite 30. s.ultat ist leider in diesem Falle ein negatives gewesen, denn wie unser Dia $\mathrm{g} r$ a $\mathrm{m} \mathrm{m}$ (Ordinaten: Konzentration der noch unzersetzten Camphocarbonsäure, Abszissen: Zeit) innerhalb der unvermeidlichen Versuchsfehler zeigt, zersetzt sich das d- und das 1-Molekül der Säure in d-Limonen als Lösungsmittel mit gleicher Geschwindigkeit und ebenso schnell auch das d- und das 1-Molekül derselben in 1-Limonen. Ich gebe aber trotz. dem die Hoffnung nicht auf, solche Fälle zu finden, wo ein optisch-aktiver Katalysator oder speziell ein optisch-aktives Lösungsmittel die Reaktionsge-

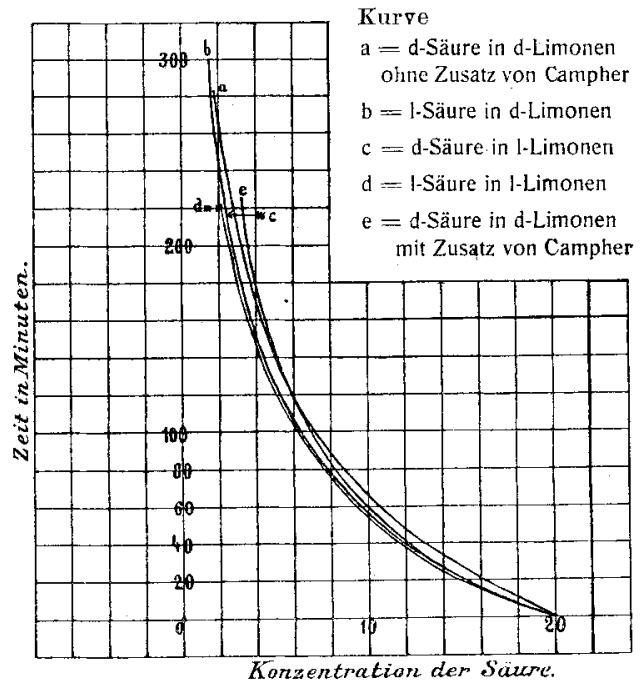

schwindigkeit der d-Molekülform des Substrates (ev. unter Zwischenreaktionen und Zwischenverbindungen) anders beeinflußt als die der I-Molekülform. Fälle, die diesem Typ schon nahe kommen ${ }^{13}$ ), sind in neuerer Zeit beobachtet, und ich werde jedem Fachgenossen für entsprechende Hinweise auf solcheoder ähnliche, kinetisch meBbare Fälle dankbar sein.

$\mathrm{R}$ aschig wird nun glauben, seine Spekulationen seien mit dem oben ausgeführten Gedanken identisch. Er sieht jedenfalls, daß ich nicht, wie er mir zuschreibt, der Ansicht bin, ,s ow i e man von Formänderung der Moleküle spreche, komme man zu unfruchtbaren Spekulationen". Das ,s o w i e" stammt von $\mathrm{R}$ a schig, nicht von mir; ich behaupte aber, es kommt sehr darauf an, wie man von der Formänderung der Moleküle spricht, und vor allem w i e man experimentell an eine solche Frage herangeht, ob man mit exakten, experimentellen, quantitativ kontrollierbaren Methoden die Natur ,,behorcht" (wie von Ba eyer sagt), was F. Raschig auf physikalisch-chemischem Gebiete merkwürdigerweise ,gerechnet" nennen würde, oder mit den allgemeinen Sentenzen und Scheinerklärungen der,gefühls mäBigen“" Ch e mie und $P$ seudoatomis tik. ,Den wundervollen Instinkt des Chemikers", um mit v a $\mathbf{n}$ "t $\mathrm{H}$ of $\mathrm{f} \mathbf{z u}$ reden (Vorlesungen III, 136), und seine Phantasie

13) Vgl. die Literatur hierüber bei : W. M e $\mathbf{y}$ e $\mathbf{r}$ h o f f e r. Gleichgewicht der Stereomeren (Leipzig 1906), Seite 63-64. 
verehre auch ich, sie dürfen aber nicht als Deckmantel für allerhand Unklarheiten dienen. Da ich durchaus nicht Raschigs Person, sonder nur seine Denkmethoden bekämpft habe, so werde ich gewiß einer der ersten sein, der Raschig zujubelt, wenn es ihm gelingen sollte, in der Tat für die Katalyse ein $\mathrm{K}$ ekulé, mit dem er sich vergleicht (diese Z. 19, 2086 [1906]), zu werden. Bis dahin aber sollte er nicht den Anspruch erheben, mit Hilfe der von mir charakterisierten Spekulationen ,unter Blinden als Einäugiger König" zu sein, sondern lieber ruhig nach dem $K$ antschen Rezepte arbeiten, ,durch sichere Erforschungen die Regeln aufzusuchen, nach welchen gewisse Erscheinungen der Natur vorgehen".

\section{Nochmals die Elektroanalyse der Metalle.}

\section{Von F. Forrstel.}

(Eingeg. d. 29.1, 1906.)

Vor kurzem habe ich dargetan 1 ), daß für die elektromalytische Metallabscheidung die Sulfat lösungen die theoretisch am übersichtlichsten sich verhaltenden Elektrolyten bilden, die vielfach vorgeschlagenen Komplexsalze aber sehr ]eicht Komplikationen hervorrufen, und $\mathrm{da} B$ in vielen praktisch gerade sehr wichtigen Fällen der Metallelektroanalyse die Anwendung der letzteren leicht vermieden und die theoretisch einfachsten Verhältnisse mit sicherem Erfolge innegehalten werden können, wenn man sich geeignet gestalteter $\mathrm{Ka}$ thoden, wie der Winklerschen Drahtnetzeloktroden, bedient. Diese Ausführungen hat $A$. Fischer ${ }^{2}$ ) einer abfälligen Kritik unterworfen, freilich nicht dadurch, daß er auch nur versucht hätte, den Kern meiner Ausführungen zu widerlegen, sondern nur, indem er, neben Angriffen auf einzelne Punkte meiner Darlegungen, darzutun sucht, daß die Komplexsalze in gewissen Fällen auch Vorteile böten, eine Auffassung, der ich freilich gar nicht entgegengetreten bia. Da bei den der Sache Fernstehenden die ron A. Fischer gegen meine Arbeit gerichteten Bemängelungen an der Triftigkeit meiner Ausführungen Bedenken erwecken könnten, will ich wenigstens dea Hauptpunkten der Kritik Fischers etwas näher treten.

Wenn er enttäuscht war, daß die meinen eingehenden theoretischen Darlegungen folgende Beschreibung von Einzelverfahren sich nur auf wenige Metalle beschränkte, so liegt das nur an seiner von mir nicht veranlaßten irrigen Erwartung, als hätte ich allerlei der verschiedensten Möglichkeiten der Metallanalyse in den Kreis meiner Untersuchungen ziehen wollen, während mir nur daran lag, in einigen, freilich in der praktischen Elektroanalyse zumeist benutzten Fällen die tatsächliche Anwondbarkeit meiner Ủberlegungen darzutun. Wenn ich dabei dio Sulfatlösungen als die die eiafachsten Verhältnisse bietenden Eilektrolyte vor-

1) Diese Zeitschr. 19, Heft 44 u. 45 (1906).

2) Diese Zeitschr. 20, Heft 4 (1907). schlug, so habe ich nirgends beansprucht, damit etwas ganz Neues gefunden zu haben, und $A$. $\mathrm{F}$ is e h e r hätte es sich wahrlich ersparen kömnen, mich darüber zu belehren, daß die Sulfatlösungea für die Elektroanalyse die zuerst angewandten Elektrolyte waren; ist es doch der. Kernpunkt meiner Ausführungen, daß man aus der Elektroanalyse alle durch die Einführung der Komplexsalze später hineingebraehten Kompli kationen überall da entfernen soll, wo sie nichtnötig sind. Die Fălle, für welche das zutrifft, sind gerade solche von aus gedehnter praktischer Bedeutung; auf sie habe ich aufmerksam gemacht.

A. Fis cher bemängelt auch meine Fmpfehlungen der Sulfatlösungen überhaupt. Er sagt: „Wenn nun die Fällung des Cadmiums aus relativ stark schwefelsaurer Lösung, dank der günstigen Beeinflussung durch die hohe Überspannung des Wasserstoffes an diesem Metall so glatt gelingt, braucht man daraus nicht notwendig zu folgern, daß man nun prinzipiell a 11 e Metallfällungen aus dieser Lösung vornehmen müsse, bzw. ausführen könne. Dies verlangt jedoch J. Foerster, ohne jedoch darauf hinzuweisen, da 3 diese Forderung mit den chemischen Eigentümlichkeiten vieler für die Elektroanalyse wichtigen Metalle unvereinbar ist. Hierher gehören Quecksilber, Blei und Wismut, Zinn und Antimon, Silber und Gold, Eisen und Mangan".

Ohne in eine nähere Erörterung der behaupteten Wichtigkeit von Gold und Silber, Eisen und Mangan für die Elektroanalyse einzutreten, führe ich hier zwei Sätze an, welche sish in Sperrdruck in meinem Aufsatze finden, von denen ich jetzt nur einige Worte besonders hervorhebe. Ich sagte einerseits: „Die praktis ch für quantitative Fällung auf der Kathode $\mathrm{m}$ eis $\mathrm{t}$ in Frage kommenden Metalle: Quecksilber, Kupfer, Cadmium, Kobalt, Nickel, Zink können also der Theorie nach aus einer leicht herstellbaren Lösung eines ihrer einfachen Salze - mit Ausnahme des aus salz. saurer Lösung zu fällenden Quecksilbers — stets aus ihrer Sulfatlösung - quantitativ durch Elektrolyse abgeschieden werden, wenn man nur die $\mathrm{H}^{-}$-Konzentration in der Lösung der Natur des zu bestimmenden Metalles anpaßt". Und später sage ich : „daß man die sauren oder alkalisehen Lösungen der Sulfate als Elektrolyte für die Elektroanalyse möglichst bevorzugen solle". A. $\mathrm{F}$ is c h e $\mathrm{r}$ hätte also obige Sätze besser vermieden.

Wenn er ferner meiner Empfehlung der Kupferfällung aus Sulfatlösung unter anderem entgegenhält, daß größere Mengen Blei vom Kupfer durch Elektrolyse nur in salpetersaurer Lösung zu trennen sind, so frage ich, ob man deshalb auch überall da, wo sehr kleine Bleimengen anwesend sind (wie bei dea meisten Messingen), oder dieses Metall ganz abwesend ist, die durch die Benutzung der Salpetersäure gegebenen, von Herrn $\mathbf{F}$ is c h e r auch gar nicht in Abrede gestellten Unbequemlichkeiten und Unübersichtlichkeiten in Kauf nehmen solle. Man war eben bisher vielfach der Meinung, daß die Anwendung salpetersaurer Lösung durch die Natur der elektrolytischen $\mathrm{K}$ a p f e r fällung erfordert werde. Dem bin ich entgegengetreten.

Was soll man aber dazu sagen, wenn $A$. 